\title{
Zonotopic Extended Kalman Filter and Fault Detection of Discrete-time Nonlinear Systems applied to a Quadrotor Helicopter
}

\author{
Ye Wang ${ }^{1}$ and Vicenç Puig ${ }^{1}$
}

\begin{abstract}
This paper presents the extension of the zonotopic Kalman filter of linear systems to nonlinear systems subject to unknown-but-bounded system disturbances and Gaussian white noises known as zonotopic extended Kalman filter (ZEKF). Due to the computational simplicity of zonotopes, the uncertain system states are bounded into a zonotopic set. The system consistency test with the system measurements is completed without using the intersection between two sets but following the extended Kalman filtering procedure that requires to find the optimal observer gain. A fault detection method based on the ZEKF algorithm is also introduced. Finally, the proposed ZEKF algorithm is applied to the quadrotor helicopter under an on-line closed-loop simulation scheme and the simulation results illustrate the effectiveness of the proposed algorithm and fault detection method.
\end{abstract}

\section{INTRODUCTION}

Kalman filter is one of the most popular technique for state estimation of linear systems in a stochastic way [1], [2], which uses the real-time measurement data including stochastic noises to update the prediction values and subsequently provide a more accurate estimated state. Kalman filter has been widely applied to numerous fields, such as robotics [3] and other real-time applications [4]. In terms of nonlinear systems, extended Kalman filter [5] and unscented Kalman filter [6] have been developed. For the extended Kalman filter, the mean estimation can be achieved by means of nonlinear functions directly and the Jacobian matrix of nonlinear system functions is used for computing the covariance of estimations. At each estimation step, the Jacobian matrix is evaluated by the current predicted system states. For the unscented Kalman filter, mean and covariance are propagated through nonlinear transformations.

Alternatively to the stochastic approach that relies on the assumption of probability distribution of noises/disturbances, the deterministic approach for state estimation using the setmembership approach [7], considers unknown-but-bounded disturbances and only the bounds of disturbances are required. In terms of the set-membership estimation approach, there are several geometric sets that can be utilized, such as polyhedrons/polytopes, ellipsoids and zonotopes. In [8], [9] and [10], the set-membership estimation approach has been well-discussed based on zonotopes due to its simple computational complexity during the state propagations. In the zonotopic state estimation, the consistency of the predicted uncertain state set is tested by checking the consistency with

\footnotetext{
${ }^{1}$ Y. Wang and V. Puig are with Advanced Control Systems (SAC) Research Group at Institut de Robòtica i Informàtica Industrial, CSIC-UPC, Universitat Politècnica de Catalunya-BarcelonaTech, C/. Llorens i Artigas 4-6. 08028 Barcelona, Spain. E-mail: ywangliri.upc.edu, vicenc.puig@upc.edu
}

the measurement state set as proposed in [9] through the intersection between two sets. However, the intersection set is not any more a zonotope and it is subsequently bounded by an over-estimation set.

In [11], a novel zonotopic Kalman filter for linear time/parameter-varying (LTV/LPV) systems has been proposed by combing the standard Kalman filtering procedure and the zonotopic state estimation. Unlike the aforementioned zonotopic state estimation approach, no intersection is required. The update of the predicted state set is based on the Kalman filter using an observer scheme. An optimal Kalman filter gain can be found, which is related to the zonotopic set and covariance matrices. In [12], both deterministic (bounded) and stochastic (Gaussian) disturbances have been taken into account. The explicit zonotopic Kalman observer is designed for two kinds of disturbances.

The quadrotor helicopter is one of popular unmanned aerial vehicles and has also attracted a lot of attention in several research fields. The dynamic model of the quadrotor helicopter is usually constructed by means of the NewtonEuler method, where the forces and torques generated from four propellers are considered. Therefore, this dynamic system is inherently unstable and it requires a proper feedback control strategy to allow its operation, such as PID control [13] and model predictive control (MPC) [14]. For the feedback control, it is necessary to obtain the current system information and measured through sensors and a system state observer can translate the partial information to estimate the total current states.

This paper extends the zonotopic Kalman filter for linear systems to nonlinear systems based on the extended Kalman filter. In fact, the extended Kalman filter use linearized partial nonlinear model. Therefore, the Jacobian matrices verified by the current system states can be obtained. Hence, the zonotopic extended Kalman filter (ZEKF) makes use of the nonlinear model and Jacobian matrices to estimate states following the linear Kalman filter procedure. As introduced [15], the fault detection method is built based on the interval observer approach. In this paper, the interval observer is built by using the ZEKF algorithm and a fault detection method is introduced as well. The application of the quadrotor helicopter is provided in order to test the effectiveness of the zonotopic extended Kalman filter. The control inputs are obtained by using a nonlinear MPC (NMPC) controller coupled to a high-fidelity simulator.

The remainder of this paper is organized as follows. Some preliminary results are briefly introduced in Section II. Problem statement is addressed in Section III. In Section 
IV, the ZEKF algorithm is provided. A fault detection based on the ZEKF algorithm for nonlinear systems is introduced in Section VII. An application of a quadrotor helicopter is provided in Section VI. Finally, conclusions are drawn in Section VII.

\section{PRELIMINARIES}

The Minkowski sum of two sets $S_{1}$ and $S_{2}$ is denoted by $S_{1} \oplus S_{2}=\left\{s_{1}+s_{2} \mid s_{1} \in S_{1}, s_{2} \in S_{2}\right\}$. The linear transformation of a set $S \subseteq \mathbb{R}^{n}$ by a vector or matrix $L \in \mathbb{R}^{r \times n}$ is denoted by $L \otimes S=\{L s \mid s \in S\}$. An interval $[a, b]$ is defined as the set $\{z \mid a \leq z \leq b\}$. The unitary interval $\mathbb{B}^{1}$ is defined as $\mathbb{B}^{1}=[-1,1]$. A unitary box $\mathbb{B}^{r} \in \mathbb{R}^{r}$ is a box composed of $r$ unitary intervals.

A zonotope $\mathcal{Z}$ is defined with its center $p \in \mathbb{R}^{n}$ and generator matrix $H \in \mathbb{R}^{n \times r}$ in a linear affine image as

$$
\mathcal{Z} \triangleq\langle p, H\rangle=p \oplus H \mathbb{B}^{r} .
$$

The Frobenius radius $J_{F}$ of $\langle p, H\rangle$ [12] is defined by the Frobenius norm of $H$ as

$$
J_{F}=\sqrt{\operatorname{tr}\left(H^{T} H\right)}=\sqrt{\operatorname{tr}\left(H H^{T}\right)},
$$

where $\operatorname{tr}(\cdot)$ is the trace of a matrix. The Frobenius radius of a vector $\mathbf{x} \sim \mathcal{Z}=\langle p, H\rangle$ is $\operatorname{tr}(\operatorname{cov}(\mathbf{x}))$, where cov denotes the covariance. In fact, the Frobenius radius can be regarded as a size criterion of a zonotope [12].

Zonotope is also a special class of geometrical set and has a flexible computational rules, some of which are formulated as follow:

$$
\begin{aligned}
\left\langle p_{1}, H_{1}\right\rangle \oplus\left\langle p_{2}, H_{2}\right\rangle & =\left\langle p_{1}+p_{2},\left[H_{1} H_{2}\right]\right\rangle \\
L \otimes\langle p, H\rangle & =\langle L p, L H\rangle .
\end{aligned}
$$

Consider a zonotope in (1), the smallest box (interval hull) containing the zonotope can be $\square \mathcal{Z}=p \oplus \operatorname{rs}(H) \mathbf{B}^{m}$, where $\operatorname{rs}(H)$ is a diagonal matrix such that $r s(H)_{i, j}=$ $\sum_{j=1}^{m}\left|H_{i, j}\right|$. Therefore, a zonotope can be enclosed within its interval hull as $\mathcal{Z} \subset \square \mathcal{Z}$.

A reduction operator for zonotopes proposed in [16] is denoted as $\downarrow_{q}$, where $q$ specifies the maximum number of column of generator matrix $H$ after reduction. Thus, $\downarrow_{q}(H)$ is computed as follows:

- Sort the column of generator matrix $H$ with the decreasing order of the Frobenius norm $\|\cdot\|$

$$
\downarrow H=\left[h_{1}, h_{2}, \ldots, h_{m}\right], \quad\left\|h_{j}\right\|^{2} \geq\left\|h_{j+1}\right\|^{2} .
$$

- Hold with first $q$-column of $\downarrow H$ and enclose the set $H_{<}$generated by remaining columns into a smallest box (interval hull)

$$
\begin{aligned}
& \text { If } m \leq q \text { then } \downarrow_{q} H=\downarrow H, \\
& \text { Else } \downarrow_{q} H=\left[H_{>}, r s\left(H_{<}\right)\right] \in \mathbb{R}^{n \times q}, \\
& H_{>}=\left[h_{1}, \ldots, h_{q}\right], H_{<}=\left[h_{q+1}, \ldots, h_{m}\right],
\end{aligned}
$$

where $r s(\cdot)$ denotes the row-sum of a matrix.

\section{Problem Statement}

Consider the discrete-time uncertain nonlinear system with additive disturbances and noises as

$$
\begin{aligned}
\mathbf{x}_{k+1} & =f\left(\mathbf{x}_{k}, \mathbf{u}_{k}\right)+E_{k} \mathbf{w}_{k}, \\
\mathbf{y}_{k} & =h\left(\mathbf{x}_{k}\right)+F_{k} \mathbf{v}_{k},
\end{aligned}
$$

where $\mathbf{x}_{k} \in \mathbb{R}^{n_{x}}, \mathbf{u}_{k} \in \mathbb{R}^{n_{u}}$ and $\mathbf{y}_{k} \in \mathbb{R}^{n_{y}}$ denote the system state, control inputs and measured outputs, respectively. $f(\cdot)$ and $h(\cdot)$ represent sequences of the nonlinear system dynamic functions and measurement functions. $E_{k}$ and $F_{k}$ are time-varying matrices of appropriate dimensions. $\mathbf{w}_{k}$ and $\mathbf{v}_{k}$ denote unknown-but-bounded additive disturbances and measurement noises, both of which are characterized as standard normal distribution in the following:

$$
\mathbf{w}_{k} \sim \mathcal{N}\left(0, I_{n_{w}}\right), \quad \mathbf{v}_{k} \sim \mathcal{N}\left(0, I_{n_{v}}\right),
$$

in which $I_{n_{w}}$ and $I_{n_{v}}$ are identity matrices. Meanwhile, $\mathbf{w}_{k}$ and $\mathbf{v}_{k}$ can be also bounded by unit hypercubes as centered zonotopes. The initial uncertain state is assumed to be in a zonotope defined by $\mathbf{x}_{0} \in \mathcal{X}_{0}=\left\langle p_{0}, H_{0}\right\rangle$.

In this paper, the objective is to find a sequence of zonotopes $\mathcal{X}_{k}$ for $\forall k \in \mathbb{Z}_{[1, N]}$ of observed system states along the horizon of $N$ through a Kalman filter-like procedure. The consistency test with system measurements is done by means of the Kalman filter.

\section{The ZEKF Algorithm of Discrete-time NONLINEAR SYSTEMS}

\section{A. Zonotopic Observer Design}

Given the discrete-time nonlinear system in (4), the standard Luenberger observer can be formulated with a timevarying observer gain $G_{k}$ as

$$
\hat{\mathbf{x}}_{k+1}=f\left(\hat{\mathbf{x}}_{k}, \mathbf{u}_{k}\right)+E_{k} \mathbf{w}_{k}+G_{k}\left(\mathbf{y}_{k}-h\left(\hat{\mathbf{x}}_{k}\right)-F_{k} \mathbf{v}_{k}\right),
$$

where $\hat{\mathbf{x}}_{k} \in \mathbb{R}^{n_{x}}$ denotes the observed states.

Based on the observer structure discussed in [12] and [11], the zonotopic nonlinear observer is introduced in the following theorem.

Theorem 1 (Nonlinear Observer Structure): Given the nonlinear system defined in (4) with initial state $\mathbf{x}_{0}$ in the zonotope $\mathcal{X}_{0}$, from the current observed state $\hat{\mathbf{x}}_{k} \in\left\langle p_{k}, H_{k}\right\rangle$, then the center and generator matrix of $\hat{\mathbf{x}}_{k+1} \in\left\langle p_{k+1}, H_{k+1}\right\rangle$ can be computed as follows:

$$
\begin{aligned}
p_{k+1} & =f\left(p_{k}, u_{k}\right)+G_{k} \mathbf{y}_{k}-G_{k} h\left(p_{k}\right), \\
H_{k+1} & =\left[\begin{array}{lll}
\left(A_{k}-G_{k} C_{k}\right) \bar{H}_{k} & E_{k} & -G_{k} F_{k}
\end{array}\right],
\end{aligned}
$$

with

$$
\begin{gathered}
A_{k}=\left.\frac{d f}{d \mathbf{x}}\right|_{\mathbf{x}=\mathbf{x}_{k}, \mathbf{u}=\mathbf{u}_{k}}=\left[\begin{array}{ccc}
\frac{\partial f_{1}}{\partial x_{1}} & \cdots & \frac{\partial f_{1}}{\partial x_{n_{x}}} \\
\vdots & \ddots & \vdots \\
\frac{\partial f_{n_{x}}}{\partial x_{1}} & \cdots & \frac{\partial f_{n_{x}}}{\partial x_{n_{x}}}
\end{array}\right]_{\mathbf{x}=\mathbf{x}_{k}, \mathbf{u}=\mathbf{u}_{k}} \\
C_{k}=\left.\frac{d h}{d \mathbf{x}}\right|_{\mathbf{x}=\mathbf{x}_{k}}=\left[\begin{array}{ccc}
\frac{\partial h_{1}}{\partial x_{1}} & \cdots & \frac{\partial h_{1}}{\partial x_{n_{x}}} \\
\vdots & \ddots & \vdots \\
\frac{\partial h_{n_{y}}}{\partial x_{1}} & \cdots & \frac{\partial h_{n_{y}}}{\partial x_{n_{x}}}
\end{array}\right]_{\mathbf{x}=\mathbf{x}_{k}}
\end{gathered}
$$




$$
\bar{H}_{k}=\downarrow_{q}\left(H_{k}\right)
$$

for $\forall k \in \mathbb{Z}_{\geq 1}$, such that $\mathcal{X}_{k+1}=\left\langle p_{k+1}, H_{k+1}\right\rangle$.

Proof: Assume $\hat{\mathbf{x}}_{k} \in\left\langle p_{k}, H_{k}\right\rangle$ is the prior condition at the time instant $k$ and by the reduction operator $\downarrow_{q}(\cdot)$, $\hat{\mathbf{x}}_{k} \in\left\langle p_{k}, \bar{H}_{k}\right\rangle$ is obtained. Following the proof procedure in Proposition 1 [11] and substituting the system matrix $A$ and $B$ with the nonlinear function $f(\cdot)$, (6a) can be obtained. Besides, (6b) is derived from the extended Kalman filter [5] and the verified Jacobian matrices $A_{k}$ and $C_{k}$ can represent the system matrices.

The ZEKF observer is defined by (5), (6a) and (6b) and depends on the a time-varying observer gain matrix $G_{k}$. Then, the estimated state $\hat{\mathbf{x}}_{k+1}$ is inside the zonotopic set denoted by $\hat{\mathbf{x}}_{k+1} \in\left\langle p_{k+1}, H_{k+1}\right\rangle$.

\section{B. Optimal Observer Gain}

Following the proposed way to compute the observer gain in [11] and [12], the optimal observer gain $G_{k}^{*}$ at time instant $k$ can be found by minimizing the Frobenius radius of the zonotope $\left\langle p_{k+1}, H_{k+1}\right\rangle$.

Theorem 2 (Optimal Nonlinear Observer Gain): Given the observer with the structure defined in Theorem 1 and the weight matrix $W=W^{T} \succ 0$, the optimal time-varying observer gain $G_{k}^{*}$ is obtained as follows

$$
\begin{aligned}
G_{k}^{*} & =A_{k} K_{k}^{*}, \\
K_{k}^{*} & =L_{k} S_{k}^{-1}, \\
L_{k} & =\bar{P}_{k} C_{k}^{T}, \\
S_{k} & =C_{k} \bar{P}_{k} C_{k}^{T}+Q_{v},
\end{aligned}
$$

where

$$
\bar{P}_{k}=\bar{H}_{k} \bar{H}_{k}^{T}, Q_{v_{k}}=F_{k} F_{k}^{T}
$$

Proof: In order to prove the above theorem, some matrices are defined as follows:

$$
P_{k+1}=H_{k+1} H_{k+1}^{T}, Q_{w_{k}}=E_{k} E_{k}^{T} .
$$

The optimal observer gain $G_{k}^{*}$ is obtained by satisfying $G_{k}=G_{k}^{*}$ such that $\frac{\partial J_{F_{k}}}{\partial G_{k}}=0$, where $J_{F_{k}}=\operatorname{tr}\left(P_{k+1}\right)$ with

$$
\begin{aligned}
P_{k+1}= & H_{k+1} H_{k+1}^{T} \\
= & \left(A_{k}-G_{k} C_{k}\right) \bar{P}_{k}\left(A_{k}-G_{k} C_{k}\right)^{T} \\
& +Q_{w_{k}}+G_{k} Q_{v_{k}} G_{k}^{T}
\end{aligned}
$$

As proved in Theorem 5 of [11], $\frac{\partial J_{F_{k}}}{\partial G_{k}}=0$ gives $\frac{\partial\left(\operatorname{tr}\left(G_{k} S_{k} G_{k}^{T}\right)\right)}{\partial G_{k}}=2 \frac{\partial \operatorname{tr}\left(A_{k} L_{k} G_{k}^{T}\right)}{\partial G_{k}}$ and it is equivalent to $\left(A_{k} L_{k}\right)^{T}=S_{k} T G_{k}^{T}$. Hence, the proof is completed.

\section{The ZEKF Algorithm}

Consider the discrete-time uncertain nonlinear system in (4), the ZEKF algorithm is based on an explicit iterations of (5) with the time-varying optimal observer gain. The observed system states at each step are bounded in a underlying zonotope computed by the ZEKF algorithm. The interval hull of the zonotope provides a deterministic interval of uncertain states. In general, the ZEKF algorithm considering $N$ simulation steps is described in Algorithm 1 .

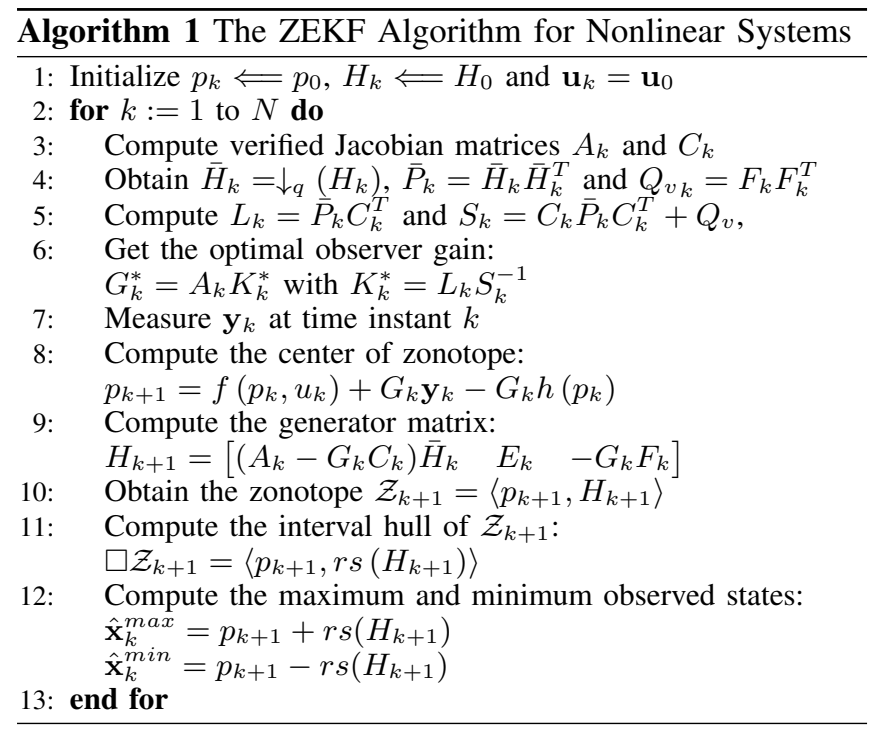

\section{Fault Detection Based on the ZEKF Algorithm}

A family of interval observers are designed by using the ZEKF algorithm in order to observe the healthy behavior of the nonlinear system and the system consistency is tested by comparing the measured outputs and healthy behaviors observed by interval observers. As defined in (5), the system states $\hat{\mathbf{x}}_{k}$ are estimated to be in the zonotope $\left\langle p_{k}, H_{k}\right\rangle$ and subsequently the healthy outputs $\hat{\mathbf{y}}_{k}$ can be also estimated to be in a zonotope $\left\langle p_{k}^{y}, H_{k}^{y}\right\rangle$, where the center $p_{k}^{y}$ and the generator matrix $H_{k}^{y}$ are described by

$$
\begin{aligned}
p_{k}^{y} & =h\left(p_{k}\right), \\
H_{k}^{y} & =\left[\begin{array}{ll}
C_{k} H_{k} & F_{k}
\end{array}\right] .
\end{aligned}
$$

The residual signals, that is the output errors, are defined by differences between the current measurements and observed outputs as follows:

$$
\mathbf{r}_{k} \triangleq \mathbf{y}_{k}-\hat{\mathbf{y}}_{k}
$$

where $\mathbf{r}_{k}$ denotes the vector of residual signals $r_{k}^{j}$ for $\forall j \in$ $\mathbb{Z}_{\left[1, n_{y}\right]}$ and $\mathbf{y}_{k}$ represents the vector of measured outputs at time instant $k$. Besides, the zonotopic set of the residual signals can be obtained since $\hat{\mathbf{y}}_{k} \in \mathcal{Y}_{k}=\left\langle p_{k}^{y}, H_{k}^{y}\right\rangle$, which is formulated as

$$
\mathcal{R}_{k} \triangleq\left\langle r_{k}^{c}, H_{k}^{r}\right\rangle=\mathbf{y}_{k} \ominus \mathcal{Y}_{k} .
$$

The consistency test can be proceed by checking whether the bounds of residual signals contain the zero vector:

$$
\mathcal{R}_{k} \in \mathbf{0},
$$

in which if the $\mathcal{R}_{k} \in \mathbf{0}$ is true, then there is no fault occurred. Otherwise, the fault is occurred at time instant $k$.

The fault detection method based on the ZEKF algorithm is proposed as follows:

Note that after fault is occurred, the zonotopic set of observed states at next simulation step is updated in Step (8) of Algorithm 1 by means of faulty measurement. Hence, a suitable fault isolation method is required. 


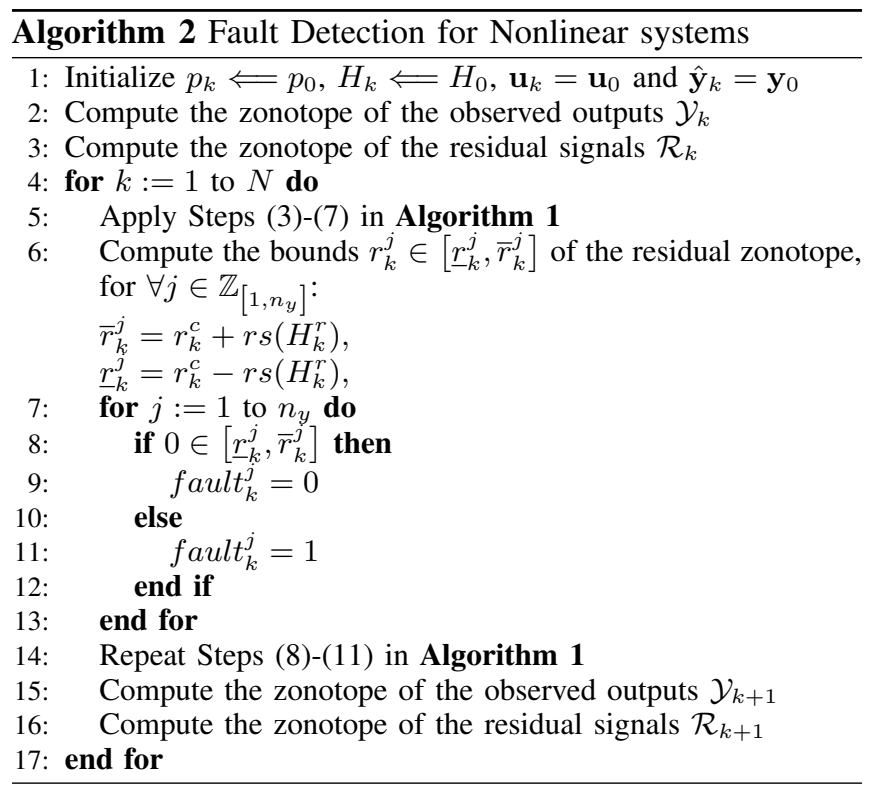

\section{Application to a Quadrotor Helicopter}

The quadrotor helicopter is configured with four propellers to produce the lift forces. The translations and rotational motions are realized by means of differences among speeds of four rotors. The dynamic model of the quadrotor helicopter uses the one proposed in [14] with mild modifications of the selection of positive directions in the inertial and body-fixed frames. The NMPC controller with full state model is utilized to provide the proper control inputs. The ZEKF algorithm is implemented in the on-line closed-loop simulations.

\section{A. Nonlinear Dynamics of the Quadrotor Helicopter}

The quadrotor helicopter has six degree-of-freedom (altitude and attitude) according to the earth-fixed frame. The altitude of this helicopter is described by $[x, y, z]^{T}$ and its attitude is defined by three Euler angles (pitch, roll and yaw) as $[\phi, \theta, \psi]^{T}$, where these three angles have to satisfy the following constraints [17]:

$$
\begin{aligned}
& -\frac{\pi}{2} \leq \phi \leq \frac{\pi}{2} \\
& -\frac{\pi}{2} \leq \theta \leq \frac{\pi}{2} \\
& -\pi \leq \psi \leq \pi
\end{aligned}
$$

Consider the earth-fixed frame as inertial frame and bodyfixed frame with origin in the mass center of the quadrotor helicopter and its body is rigid and symmetric, the continuous-time dynamic model of the quadrotor helicopter is written as follows [14]:

$$
\begin{aligned}
\ddot{\phi} & =\dot{\theta} \dot{\psi} a_{1}+\dot{\theta} a_{2} \Omega_{r}+b_{1} U_{2}, \\
\ddot{\theta} & =\dot{\phi} \dot{\psi} a_{3}-\dot{\phi} a_{4} \Omega_{r}+b_{2} U_{3}, \\
\ddot{\psi} & =\dot{\phi} \dot{\theta} a_{5}+b_{3} U_{4}, \\
\ddot{z} & =-g+(\cos \phi \cos \theta) U_{1} / m, \\
\ddot{x} & =(\cos \phi \sin \theta \cos \psi+\sin \phi \sin \psi) U_{1} / m, \\
\ddot{y} & =(\cos \phi \sin \theta \sin \psi-\sin \phi \cos \psi) U_{1} / m,
\end{aligned}
$$

with

$$
\begin{aligned}
& {\left[\begin{array}{l}
U_{1} \\
U_{2} \\
U_{3} \\
U_{4} \\
\Omega_{r}
\end{array}\right]=\left[\begin{array}{c}
b\left(\Omega_{1}^{2}+\Omega_{2}^{2}+\Omega_{3}^{2}+\Omega_{4}^{2}\right) \\
b\left(\Omega_{2}^{2}-\Omega_{4}^{2}\right) \\
b\left(-\Omega_{1}^{2}+\Omega_{3}^{2}\right) \\
d\left(-\Omega_{1}^{2}+\Omega_{2}^{2}-\Omega_{3}^{2}+\Omega_{4}^{2}\right) \\
-\Omega_{1}+\Omega_{2}-\Omega_{3}+\Omega_{4}
\end{array}\right]} \\
& a_{1}=\frac{I_{y y}-I_{z z}}{I_{x x}}, a_{2}=\frac{J_{r}}{I_{x x}}, a_{3}=\frac{I_{z z}-I_{x x}}{I_{y y}}, \\
& a_{4}=\frac{J_{r}}{I_{y y}}, a_{5}=\frac{I_{x x}-I_{y y}}{I_{z z}}, \\
& b_{1}=\frac{\ell}{I_{x x}}, b_{2}=\frac{\ell}{I_{y y}}, b_{3}=\frac{1}{I_{z z}} .
\end{aligned}
$$

where $\Omega_{1}, \Omega_{2}, \Omega_{3}$ and $\Omega_{4}$ denote speeds of four rotors, respectively. $I_{x x}, I_{y y}$ and $I_{z z}$ are the moments of inertial of the quadrotor helicopter for three axes, respectively. $J_{r}$ is the rotational moment of inertial. $\ell$ denotes the arm length of the quadrotor helicopter. Moreover, $b$ and $d$ represent thrust and drag coefficients.

\section{B. Nonlinear Control-oriented Model of the Quadrotor He- licopter}

According to the dynamic model of the quadrotor helicopter, from the control point of view, the vectors of system state $\mathbf{x}$, control input $\mathbf{u}$ and measured output $\mathbf{y}$ are chosen as follows:

$$
\begin{aligned}
& \mathbf{x}=\left[\begin{array}{llllllllllll}
x_{1} & x_{2} & x_{3} & x_{4} & x_{5} & x_{6} & x_{7} & x_{8} & x_{9} & x_{10} & x_{11} & x_{12}
\end{array}\right]^{T} \\
& =\left[\begin{array}{llllllllllll}
\phi & \dot{\phi} & \theta & \dot{\theta} & \psi & \dot{\psi} & z & \dot{z} & x & \dot{x} & y & \dot{y}
\end{array}\right]^{T}, \\
& \mathbf{u}=\left[\begin{array}{llll}
u_{1} & u_{2} & u_{3} & u_{4}
\end{array}\right]^{T}=\left[\begin{array}{llll}
\Omega_{1} & \Omega_{2} & \Omega_{3} & \Omega_{4}
\end{array}\right]^{T}, \\
& \mathbf{y}=\left[\begin{array}{lll}
y_{1} & y_{2} & y_{3}
\end{array}\right]^{T}=\left[\begin{array}{lll}
z & x & y
\end{array}\right]^{T} .
\end{aligned}
$$

By means of the Euler discretization method, the discretetime uncertain control-oriented model of the quadrotor helicopter in presence of state disturbances and measurement noises is generally formulated as

$$
\begin{aligned}
\mathbf{x}_{k+1} & =f\left(\mathbf{x}_{k}, \mathbf{u}_{k}\right)+E \mathbf{w}_{k} \\
\mathbf{y}_{k} & =C \mathbf{x}_{k}+F \mathbf{v}_{k}
\end{aligned}
$$


with

$$
\begin{aligned}
& E=\left[\begin{array}{cccccccccccc}
0 & 0 & 0 & 0 & 0 & 0 & 0 & 0 & 0 & 0 & 0 & 0 \\
0 & 0 & 0 & 0 & 0 & 0 & 0 & 0 & 0 & 0 & 0 & 0 \\
0 & 0 & 0 & 0 & 0 & 0 & 0 & 0 & 0 & 0 & 0 & 0 \\
0 & 0 & 0 & 0 & 0 & 0 & 0 & 0 & 0 & 0 & 0 & 0 \\
0 & 0 & 0 & 0 & 0 & 0 & 0 & 0 & 0 & 0 & 0 & 0 \\
0 & 0 & 0 & 0 & 0 & 0 & 0 & 0 & 0 & 0 & 0 & 0 \\
0 & 0 & 0 & 0 & 0 & 0 & 0 & 0 & 0 & 0 & 0 & 0 \\
0 & 0 & 0 & 0 & 0 & 0 & 0 & 0.03 & 0 & 0 & 0 & 0 \\
0 & 0 & 0 & 0 & 0 & 0 & 0 & 0 & 0 & 0 & 0 & 0 \\
0 & 0 & 0 & 0 & 0 & 0 & 0 & 0 & 0 & 0.03 & 0 & 0 \\
0 & 0 & 0 & 0 & 0 & 0 & 0 & 0 & 0 & 0 & 0 & 0 \\
0 & 0 & 0 & 0 & 0 & 0 & 0 & 0 & 0 & 0 & 0 & 0.03
\end{array}\right], \\
& C=\left[\begin{array}{llllllllllll}
0 & 0 & 0 & 0 & 0 & 0 & 1 & 0 & 0 & 0 & 0 & 0 \\
0 & 0 & 0 & 0 & 0 & 0 & 0 & 0 & 1 & 0 & 0 & 0 \\
0 & 0 & 0 & 0 & 0 & 0 & 0 & 0 & 0 & 0 & 1 & 0
\end{array}\right] \\
& F=\left[\begin{array}{cccccccccccc}
0 & 0 & 0 & 0 & 0 & 0 & 0.01 & 0 & 0 & 0 & 0 & 0 \\
0 & 0 & 0 & 0 & 0 & 0 & 0 & 0 & 0.01 & 0 & 0 & 0 \\
0 & 0 & 0 & 0 & 0 & 0 & 0 & 0 & 0 & 0 & 0.01 & 0
\end{array}\right] .
\end{aligned}
$$

where $C, E$ and $F$ are assumed as time-invariant matrices. The sensors only provide the altitude informations. System disturbances and measurement noises are assumed as Gaussian white noises following the normal distribution:

$$
\mathbf{w}_{k} \sim \mathcal{N}\left(0, I_{n_{w}}\right), \quad \mathbf{v}_{k} \sim \mathcal{N}\left(0, I_{n_{v}}\right) .
$$

\section{The Closed-loop Simulation Scheme}

Fig. 1 shows the on-line closed-loop simulation scheme. The design of the NMPC controller is out of the scope of this paper. Assume that the NMPC controller for the quadrotor helicopter can provide the admissible control inputs when the system is under perturbations and the full-state information can be fed back to the NMPC controller. The observer is designed by using the ZEKF algorithm in Algorithm 1. At each sampling time, the full states can be observed through the ZEKF observer as $\hat{\mathbf{x}}_{k}$ in a zonotopic set. The center of the zonotopic set can be regarded as the nominal observation. The bounded box (interval hull) of the zonotopic set describes the bounds of observed states including the propogated uncertainty. On the other hand, some unexpected faults (from actuators or sensors) might happen during the simulations. It is assumed that the NMPC controller has the capacity to tolerate these faults. By applying Algorithm 2, the occurred faults can be detected.

The closed-loop simulation is implemented in MATLAB R2015a (64-bit) with YALMIP toolbox [18] and IPOPT nonlinear solver included in OPTI toolbox [19].

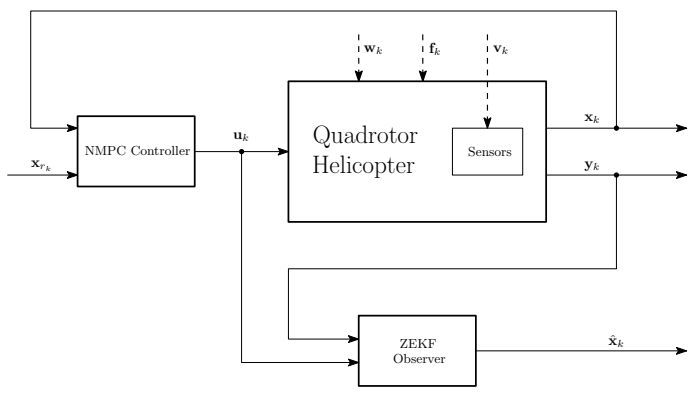

Fig. 1. The on-line closed-loop simulation scheme

\section{Results}

Fig. 2 shows the results of applying the ZEKF algorithm to the quadrotor helicopter. The bounds of observed states (blue real lines) in Fig. 2 are due to the Gaussian noises included in the measurement sensors. From all these figures, the state observations (red dashed lines) are approximating quite well the data obtained from the simulator of the quadrotor helicopter. Therefore, the ZEKF algorithm is available for estimating the full system states using system measurements obtained from the non-linear simulator.

The results of fault detection are shown in Fig. 3. Because there are three measured outputs, three residuals are generated. By checking whether the residual intervals contain zero, the occurred fault is detected in Residual 1 at time 40s. In order to discover where is the fault, a appropriate fault isolation method is required. But this paper did not include the fault isolation method. Therefore, as shown in Fig. 3(a), after the fault at time 40s is detected, the healthy interval observer is updated by the measurement including the detected fault.

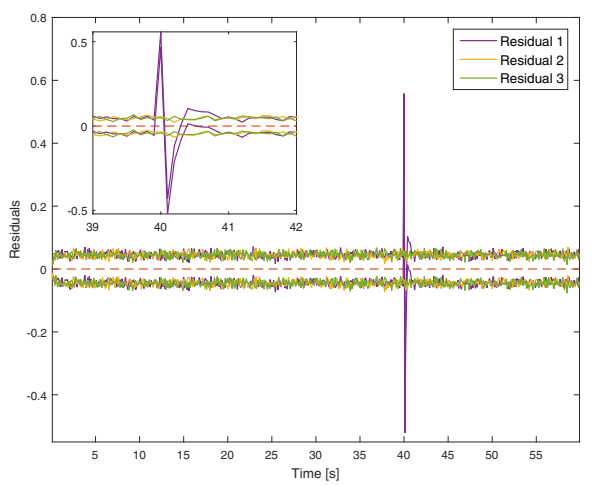

(a) Residual signals

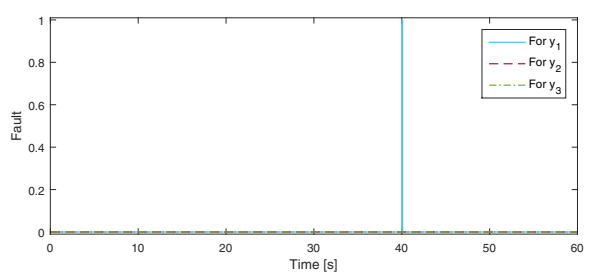

(b) Detected faults

Fig. 3. Fault detection results of the quadrotor helicopter

\section{CONCLUSIONS}

This paper extends the zonotopic Kalman filter to nonlinear system and presents the application results of applying the ZEKF algorithm to a quadrotor helicopter. The zonotopic Kalman filter for linear systems is extended into nonlinear systems under perturbations. During the on-line closed-loop simulation, the additive disturbances to system states and measurement noises are sampled by random values with known bounds. The ZEKF algorithm has been successfully applied into the quadrotor helicopter in simulation and the 


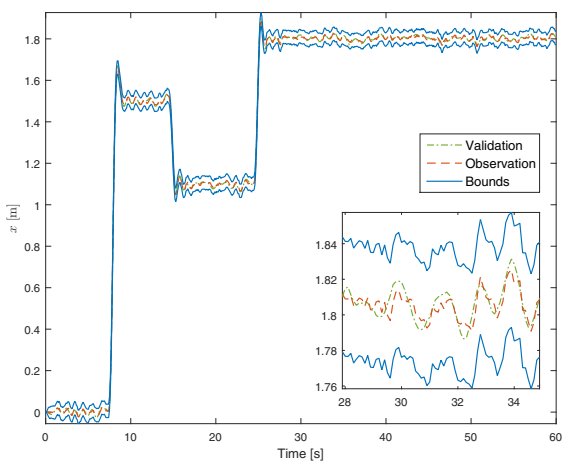

(a) $x$

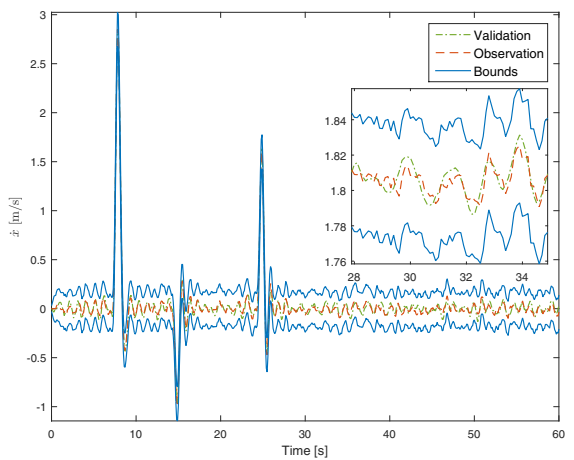

(d) $\dot{x}$

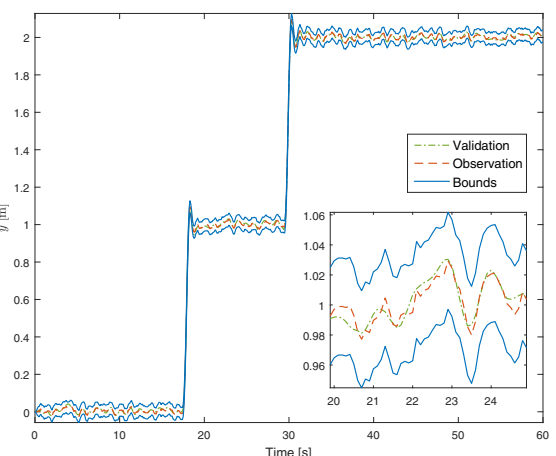

(b) $y$

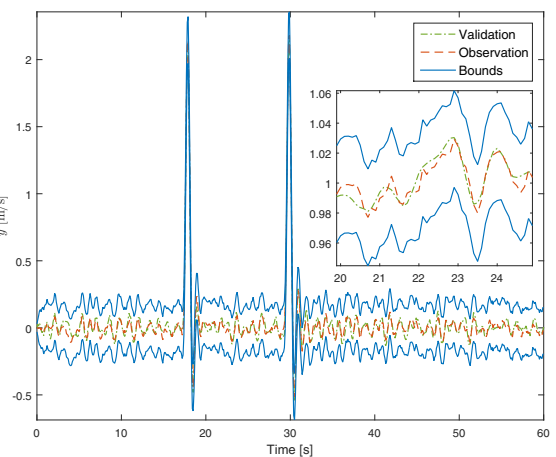

(e) $\dot{y}$

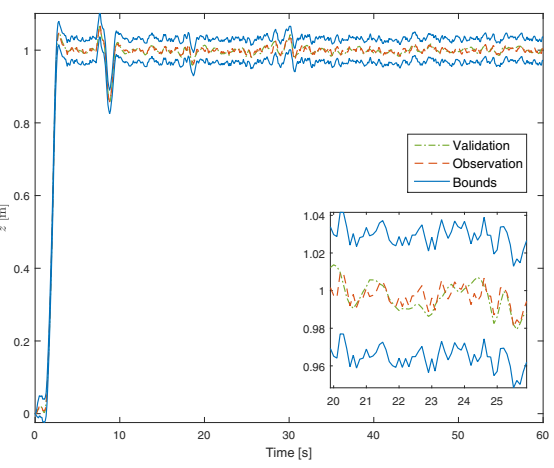

(c) $z$

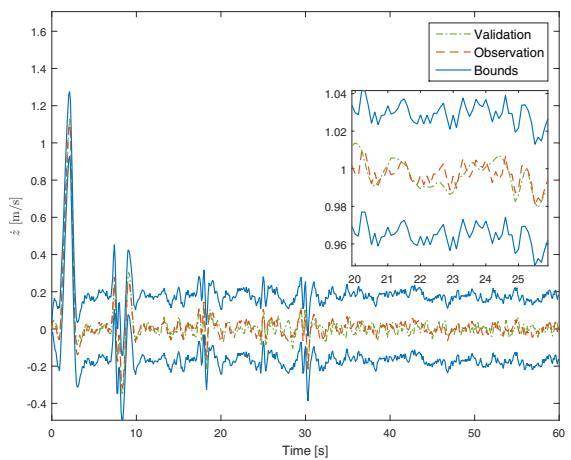

(f) $\dot{z}$

Fig. 2. Sampled observed states of the quadrotor helicopter

estimated states are in agreement with the non-linear simulated behaviour. Moreover, the faults in the system can be detected by means of the proposed fault detection method.

\section{ACKNOWLEDGMENT}

This work has been partially funded by the Spanish Government (MINECO) through the project CICYT ECOCIS (ref. DPI2013-48243-C2-1-R), by MINECO and FEDER through the project CICYT HARCRICS (ref. DPI201458104-R).

\section{REFERENCES}

[1] R. E. Kalman, "A new approach to linear filtering and prediction problems," ASME Journal of Basic Engineering”, vol. 82, no. 1, pp. $35-45,1960$.

[2] R. E. Kalman and R. S. Bucy, "New results in linear filtering and prediction theory," ASME Journal of Basic Engineering", vol. 83, no. 1 , pp. $95-108,1961$.

[3] A. Kelly, "A 3d space formulation of a navigation kalman filter for autonomous vehicles," Robotics Institute, Pittsburgh, PA, Tech. Rep. CMU-RI-TR-94-19, May 1994.

[4] C. Chui and G. Chen, Kalman Filtering with Real-Time Applications, ser. Information Sciences. New York, USA: Springer, 2009, vol. 17.

[5] S. Julier and J. Uhlmann, "New extension of the kalman filter to nonlinear systems," Proc. SPIE, vol. 3068, pp. 182 - 193, 1997.

[6] J. Simon and J. Uhlmann, "Unscented filtering and nonlinear estimation," in Proceedings of the IEEE, 2004, pp. $401-422$.

[7] F. Schweppe, "Recursive state estimation: Unknown but bounded errors and system inputs," IEEE Transactions on Automatic Control, vol. 13 , no. 1 , pp. $22-28,1968$.

[8] V. Puig, P. Cuguero, and J. Quevedo, "Worst-case state estimation and simulation of uncertain discrete-time systems using zonotopes," in European Control Conference, 2001, pp. 1691 - 1697.

[9] T. Alamo, J. Bravo, and E. Camacho, "Guaranteed state estimation by zonotopes," Automatica, vol. 41, no. 6, pp. 1035 - 1043, 2005.
[10] J. Bravo, T. Alamo, and E. Camacho, "Bounded error identification of systems with time-varying parameters," IEEE Transactions on Automatic Control, vol. 51, no. 7, pp. 1144 - 1150, 2006.

[11] C. Combastel, "Zonotopes and kalman observers: Gain optimality under distinct uncertainty paradigms and robust convergence," Automatica, vol. 55, pp. 265 - 273, 2015.

[12] _ _ "Merging kalman filtering and zonotopic state bounding for robust fault detection under noisy environment," in 9th IFAC Symposium on Fault Detection, Supervision and Safety for Technical Processes (SAFEPROCESS), vol. 48, no. 21, 2015, pp. $289-295$.

[13] K. Alexhs, G. Nikolakopoulos, and A. Tzes, "Autonomous quadrotor position and attitude pid/pidd control in gps-denied environments," International Review of Automatic Control, vol. 4, no. 3, 2011.

[14] K. Alexis, G. Nikolakopoulos, and A. Tzes, "Model predictive quadrotor control: attitude, altitude and position experimental studies," IET Control Theory Applications, vol. 6, no. 12, pp. 1812 - 1827, 2012

[15] V. Puig, J-Quevedo, T. Escobet, and S. Heras, "Passive robust fault detection approaches using interval models," in 15th IFAC World Congress, 2002, pp. $405-405$.

[16] C. Combastel, "A state bounding observer for uncertain non-linear continuous-time systems based on zonotopes," in 44th IEEE Conference on Decision and Control and European Control Conference (CDC-ECC), 2005, pp. $7228-7234$.

[17] L. Derafa, T. Madani, and A. Benallegue, "Dynamic modelling and experimental identification of four rotors helicopter parameters," in IEEE International Conference on Industrial Technology, 2006, pp. $1834-1839$.

[18] J. Löfberg, "YALMIP: A toolbox for modeling and optimization in MATLAB," Taipei, Taiwan, 2004. [Online]. Available: http://users.isy.liu.se/johanl/yalmip

[19] J. Currie and D. Wilson, "OPTI: Lowering the Barrier Between Open Source Optimizers and the Industrial MATLAB User," Savannah, Georgia, USA, 2012. 